\title{
Scanning Transmission Electron Microscopy (STEM) Study on Novel Two- dimensional Materials
}

\author{
Shi-ze Yang
}

Eyring Materials Center, Arizona State University, Tempe, Arizona, United States

Two-dimensional materials with typical characteristics of atomic layer thickness, weak inter layer Van de Walls interaction and large surface areas are emerging superstar for materials science, physics and chemistry. For example, the Nobel prize winning material graphene has demonstrated numerous promising applications ranging from photonics, catalysis, sensors, flexible electronics etc. Transition metal dichalcogenides overcome the limitation of zero bandgap in graphene, is expected to bring in new applications in nanoelectronics, single photon emitters, spintronics etc.

Aberration corrected scanning transmission electron microscopy in the annular dark field (ADF) imaging mode provides atomic level spatial resolution and $\mathrm{Z}$ number sensitive image contrast. Therefore, atomic level chemical information is feasible in the STEM-ADF images. Local atomic structures, defects and phases can be revealed in a straightforward fashion. Such microscopy results can be combined with density functional theory simulations and synchrotron X-ray absorption measurements to provide both local and bulk information to provide in-depth understanding on the physical property and performance of materials.

I will talk about how STEM technique provides a powerful tool to help understand the two-dimensional materials for applications including catalysis and electronics. Transition metal dichalcogenide materials will be the focus. I will first talk about Re doped $\mathrm{MoS}_{2}$ atomic layers [1,2]. These thin layers are very sensitive to electron beam and requires special consideration for the damage effect. I adopted low primary voltage $(60 \mathrm{kV})$ to reduce the knock-on damage. It is revealed that electron beam not only can image materials but can also trigger or manipulate the movement of single dopant atoms [1]. Atomic level chemical mapping is realized in Re doped $\mathrm{MoS}_{2}$ alloy using the ADF contrast [2]. I will also show that how electron microscopy help reveal the structures of $\mathrm{SnS}_{2}$ after transition metal intercalation [3].

Two-dimensional materials with strong layer coupling are emerging with exotic properties. Here I will show the study on $\mathrm{PdSe}_{2}$ and how the reactive ion etching resulted in the new phase of $\mathrm{Pd}_{17} \mathrm{Se}_{15}$. [4,5] Transition metal single atom catalysts show great promise in catalysis. I will use two examples to show how STEM technique not only provide direct evidence of single atoms to complement synchrotron X-ray absorptions but also reveal local bonding environment directly [6-8]. As one last example, I will show how three-dimensional coordinates of atoms in single layer Re doped $\mathrm{MoS}_{2}$ can be resolved using electron tomography [9]. I will also show some outlook for study on two-dimensional materials with monochromated electron beams.

\section{Acknowledgements:}

Most of the electron microscopy work is carried out at Oak Ridge National Laboratory and was supported by the U.S. Department of Energy, Office of Science, Basic Energy Sciences, Materials Science and Engineering Division and through a user proposal supported by ORNL's Center for Nanophase Materials Sciences, which is sponsored by the Scientific User Facilities Division of U.S. Department of Energy. Part of the work in the talk used facilities within the Eyring Materials Center at Arizona State University supported in part by NNCI-ECCS-1542160. 

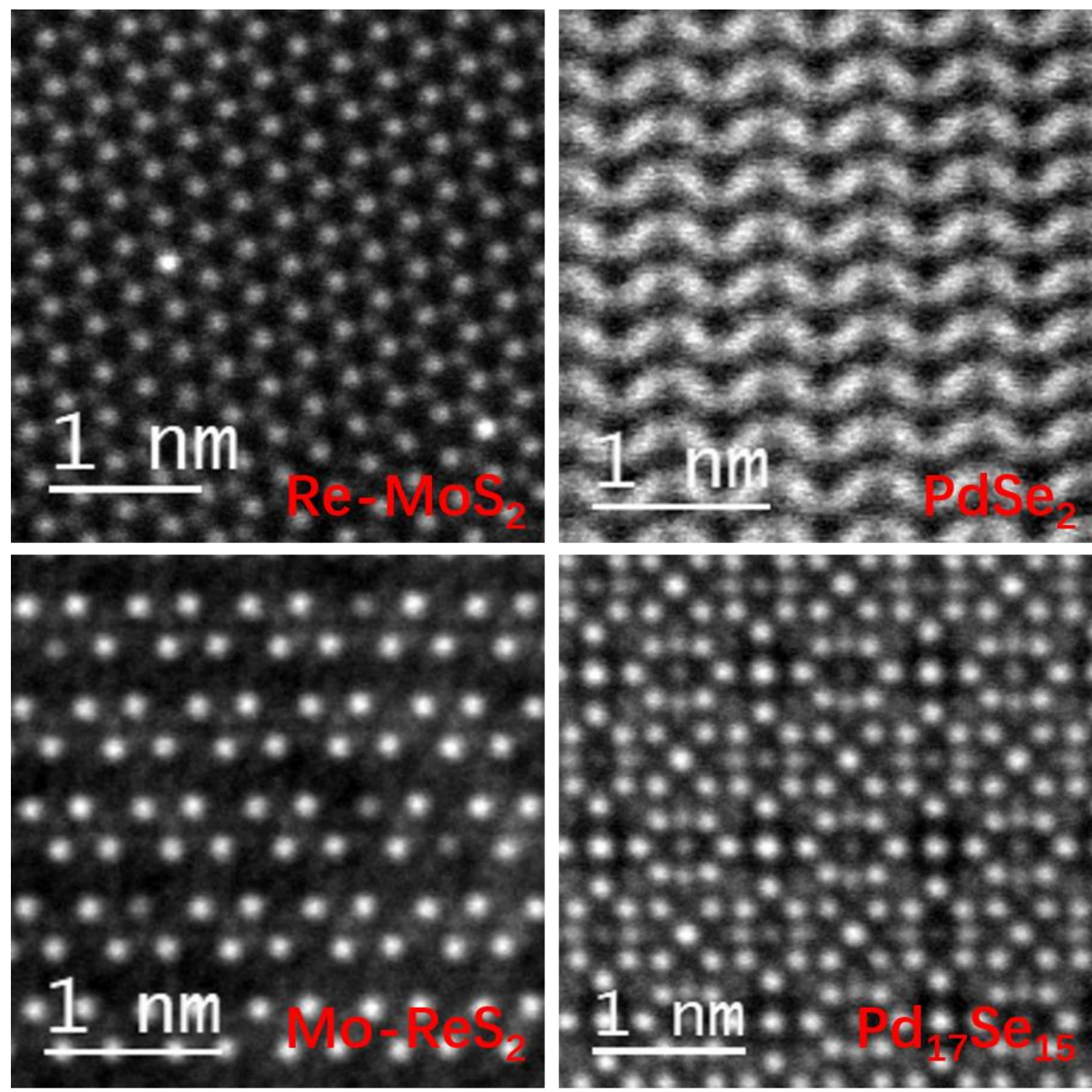

Figure 1. Various two-dimensional materials studied using STEM technique.

References

[1] Yang, Shi-Ze, Weiwei Sun, et al. "Direct cation exchange in monolayer MoS 2 via recombinationenhanced migration." Physical review letters 122, no. 10 (2019): 106101.

[2] Yang, S.Z., Gong, Y., Manchanda, P., et al., 2018. Rhenium-Doped and Stabilized MoS2 Atomic Layers with Basal-Plane Catalytic Activity. Advanced Materials, 30(51), p.1803477.

[3] Gong, Y., Yuan, H., Wu, C.L., Tang, P., Yang, S.Z., et al. Spatially controlled doping of twodimensional SnS 2 through intercalation for electronics. Nature nanotechnology, 13(4), pp.294-299.

[4] Oyedele, A.D., Yang, S. et al. PdSe2: pentagonal two-dimensional layers with high air stability for electronics. Journal of the American Chemical Society, 139(40), pp.14090-14097.

[5] Oyedele, A.D., Yang, S., et al., 2019. Defect-Mediated Phase Transformation in Anisotropic Two-

Dimensional PdSe2 Crystals for Seamless Electrical Contacts. Journal of the American Chemical Society, 141(22), pp.8928-8936. 
[6] Cheng, Y., Zhao, S., et al., 2018. Atomically dispersed transition metals on carbon nanotubes with ultrahigh loading for selective electrochemical carbon dioxide reduction. Advanced Materials, 30(13), p.1706287.

[7] Cheng, Y., Zhao, S. et al., 2019. Unsaturated edge-anchored Ni single atoms on porous microwave exfoliated graphene oxide for electrochemical CO2. Applied Catalysis B: Environmental, 243, pp.294303.

[8] Zhao, S., Chen, G., Zhou, G. et al. 2020. A Universal Seeding Strategy to Synthesize Single Atom Catalysts on 2D Materials for Electrocatalytic Applications. Advanced Functional Materials, 30(6), p.1906157.

[9] Tian, X., Kim, D.S., Yang, S., et al., 2019. Correlating 3D atomic defects and electronic properties of 2D materials with picometer precision. arXiv preprint arXiv:1901.00633. (accepted at Nature Materials) 